research highlights

NONLINEAR OPTICS

\section{Metasurface mixer}

Nat. Commun. 9, 2507 (2018)

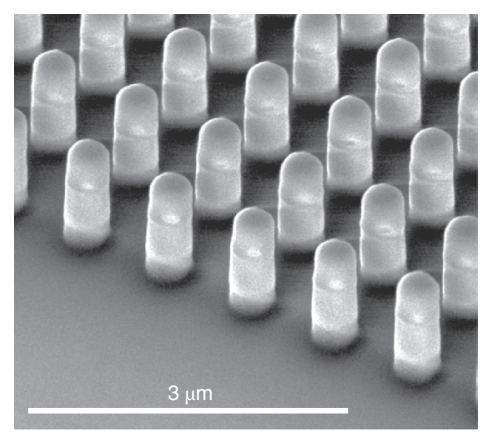

Credit: Springer Nature Ltd

Optical frequency mixers generally rely on bulk nonlinear crystals and weak nonlinear optical processes. Sheng Liu and colleagues from Sandia National Laboratories, USA have now demonstrated an ultra-compact all-dielectric metamixer that enables many simultaneous nonlinear optical processes across a broad spectral range. The device is a gallium arsenidebased metasurface consisting of an 840-nm periodic square array of nanocylinders with a diameter of $\sim 400 \mathrm{~nm}$. Each nanocylinder is composed of an $~ 300$-nm-thick $\mathrm{SiO}_{\mathrm{x}}$ etch mask, an $\sim 450$-nm-thick GaAs nanodisk and an $~ 400$-nm-thick $\left(\mathrm{Al}_{x} \mathrm{Ga}_{1-x}\right) \mathrm{O}_{3}$ layer. On pumping the metamixer with two femtosecond pulses at $\sim 1.24 \mu \mathrm{m}$ and $\sim 1.57 \mu \mathrm{m}$, near the magnetic and electric dipole resonances of the metasurface, the team observed the generation of 11 new frequencies spanning from $\sim 380 \mathrm{~nm}$ to $\sim 1,000 \mathrm{~nm}$, generated by 7 different concurrent nonlinear processes (second-harmonic, third-harmonic and fourth-harmonic generations, sumfrequency generation, two-photonabsorption-induced photoluminescence, four-wave mixing and six-wave mixing). Applications in communications, quantum optics, sensing and biology are expected.

$R W$

https://doi.org/10.1038/s41566-018-0230-4

\section{POLARITONS}

\section{Spin orientation switching} Phys. Rev. B 97, 235303 (2018)

For the realization of integrated spintronic devices, a spin-switching device is desired. Now, Alexis Askitopoulos and co-workers from the UK, Russia and Greece have experimentally observed that the two spin components of a polariton condensate can switch their orientation via illumination with light. A polariton condensate was generated in a high-quality $(Q=16,000)$ GaAs-based microcavity consisting of $10 \mathrm{~nm}$ GaAs quantum wells and a Rabi splitting of $9 \mathrm{meV}$. Light from a continuous-wave Ti:sapphire laser emitting at $754 \mathrm{~nm}$ was sent to the microcavity and the first excited state of the polariton condensate was optically trapped. The spin component of the condensate was investigated by means of polarizationresolved spectroscopy. When the excitation density of the light was increased from $12.48 \mathrm{~mW}$ to $14.56 \mathrm{~mW}$, the dominant spinor state with a $p$-orbital symmetry was rotated by $\pi / 2$. The phenomenon is explained by the spatially inhomogeneous depletion of the exciton reservoir and the repulsive exciton-polariton interaction. In principle, the approach could lead to a spin-demultiplexing device for polaritonbased spin circuits.

https://doi.org/10.1038/s41566-018-0227-z
MICROWAVE PHOTONICS

\section{Single-mode operation \\ Sci. Adv. 4, eaar6782 (2018)}

The concept of optical parity-time (PT) symmetry, whereby optical gain and loss are carefully controlled within a photonic system, has enabled the generation of pure, low-noise single-mode microwave signals without the need for complex filtering. In an optoelectronic oscillator, an optical feedback loop is used to generate high-frequency microwave signals with low phase noise, but ensuring single-mode operation usually requires the use of an ultra-narrow-band optical filter. Now, Jiejun Zhang and Jianping Yao from the University of Ottawa, Canada have shown that employing PT symmetry can remove the need for such a filter. In their approach, a polarizing beam splitter and polarization controllers are used to create two feedback loops that have a variable splitting ratio, thus allowing the level of gain and loss to be controlled and PT symmetry to be broken for just one mode, enabling single-mode operation. The approach has been tested with optical fibre loops of $20.31 \mathrm{~m}, 433.1 \mathrm{~m}$ and $9.166 \mathrm{~km}$ in length and demonstrated to produce a microwave signal at a frequency of $9.867 \mathrm{GHz}$ with a phase noise of $-142.5 \mathrm{dBc} \mathrm{Hz}^{-1}$. $\quad O G$

https://doi.org/10.1038/s41566-018-0229-X

\section{METAMATERIALS}

\section{Deep-learning boost}

ACS Nano 12, 6326-6334 (2018)

Wei Ma and colleagues from Northeastern University, USA have developed an accurate, efficient deep-learning-based model for designing three-dimensional chiral metamaterials. The model consists of two bidirectional neural networks, with a forward combiner and an inverse combiner. It is trained heuristically with multiple functions for fast prototyping, optimization and inverse design. The unit cell of the chiral metamaterial under study consists of two stacked gold splitring resonators twisted at a certain angle and separated by two spacing dielectric layers with a continuous gold reflector at the bottom. The team demonstrated that the model can significantly shorten the prediction time for generating chiral dichroism and can solve the design-on-demand inverse problem accurately and efficiently, retrieving geometric parameters of the metamaterial from specific requirements of its optical response. $\quad R W$

https://doi.org/10.1038/s41566-018-0231-3

Oliver Graydon, Noriaki Horiuchi and Rachel Won 